\title{
An $h p$ finite element adaptive scheme to solve the Laplace model for fluid-solid vibrations
}

\author{
M. G. Armentano ${ }^{\mathrm{a}}$, C. Padra ${ }^{\mathrm{b}}$, R. Rodríguez ${ }^{\mathrm{c}}$, M. Scheble ${ }^{\mathrm{b}, *}$ \\ ${ }^{a}$ Departamento de Matemática, Facultad de Ciencias Exactas y Naturales, Universidad de Buenos Aires, 1428, Buenos Aires, Argentina \\ ${ }^{b}$ Centro Atómico Bariloche, 4800, Bariloche, Argentina \\ ${ }^{c} C I^{2} M A$, Departamento de Ingeniería Matemática, Universidad de Concepción, Casilla 160-C, Concepción, Chile
}

\begin{abstract}
In this paper we introduce an $h p$ finite element method to solve a two-dimensional fluid-structure spectral problem. This problem arises from the computation of the vibration modes of a bundle of parallel tubes immersed in an incompressible fluid. We prove the convergence of the method and a priori error estimates for the eigenfunctions and the eigenvalues. We define an a posteriori error estimator of the residual type which can be computed locally from the approximate eigenpair. We show its reliability and efficiency by proving that the estimator is equivalent to the energy norm of the error up to higher order terms, the equivalence constant of the efficiency estimate being suboptimal in that it depends on the polynomial degree. We present an $h p$ adaptive algorithm and several numerical tests which show the performance of the scheme, including some numerical evidence of exponential convergence.
\end{abstract}

Keywords:

fluid structure interaction, vibration problem, finite elements, $h p$ version, spectral approximation, a posteriori error estimates

\section{Introduction}

The goal of this paper is to introduce and analyze an $h p$ finite element scheme for solving a fluid-structure interaction problem: the two dimensional Laplace model for fluid-solid vibrations.

In recent decades, the numerical approximation of spectral problems arising in fluid mechanics have received increasing attention (see $[1,2,3,4,5,6]$ and the references therein). In particular, the problem considered in this paper, which corresponds to approximating the vibrations of a bundle of tubes immersed in a fluid contained in a rigid cavity, has a considerable importance in nuclear engineering and has been studied for several authors (see, for example, $[4,7,8]$ ).

It is well known that adaptive procedures based on a posteriori error indicators play nowadays a relevant role in the numerical solution of partial differential equations. In particular, there are several papers concerning the development of a-posteriori error estimates and efficient adaptive schemes for the $h$-finite element approximation of different eigenvalue problems (see, for example, $[9,10,11,12,13])$. There are also some recent references regarding the $h p$ finite element approximation of eigenvalue problems (see, for instance, $[18,19,20]$ ). However, the bibliography about $h p$-adaptive schemes for this kind of problems is scarce and mainly focused on electromagnetics (see, for instance, [21, 22] and [23] for a survey on the application of the $h p$-finite element method to electromagnetism, including eigenvalue problems). On the other hand, the a-posteriori

\footnotetext{
${ }^{*}$ Corresponding author.

Email addresses: garmenta@dm.uba.ar (M. G. Armentano), padra@cab.cnea.gov.ar (C. Padra), rodolfo@ing-mat.udec.cl (R. Rodríguez), scheble@cab.cnea.gov.ar (M. Scheble)
}

error analysis for the $h p$ version of the finite element method still present several challenges, even for source problems (see, for instance, $[14,15,16,17]$ and the references therein). One of the main difficulties in $h p$-adaptivity arises from the fact that the accuracy can be improved in two different ways, either by subdividing elements or by increasing the polynomial degree.

In this paper we introduce and analyze an $h p$ finite element approximation of the spectral problem described above. We obtain a-priori error estimates and develop an a-posteriori error estimator of the residual type which can be computed locally from the approximate eigenpair. We analyze the equivalence of this estimator with the energy norm of the error. In particular, we prove global reliability and local efficiency estimates, both up to higher order terms, the latter with a constant which depends on the polynomial degree of the element. To the best of the authors' knowledge, simultaneous reliability and efficiency estimates, both with constants independent of the polynomial degree, have not been proved yet for any a posteriori error estimator for $h p$ finite element methods. Nevertheless, the numerical experiments suggest that the proposed error indicator points out correctly the elements with largest error. Following the $h p$ adaptive strategy given in [16], we propose an adaptive algorithm and apply it to different cavities and shapes of tubes. These numerical tests allow us to show the good performance of the error indicator and the adaptive algorithm, including an exponential rate of convergence in terms of the number of degrees of freedom.

The rest of the paper is organized as follows. In Section 2 we introduce the fluid-solid vibration problem. In Section 3 we present the $h p$ finite element approximation and obtain a-priori error estimates. In Section 4 we introduce the a posteriori error estimator and prove its equivalence with the energy norm of the 
error. In Section 5 we analyze some numerical aspects concerning the solution of the discrete generalized eigenvalue problem and introduce the adaptive refinement strategy. In Section 6 we report several numerical examples which allow assessing the performance of the adaptive scheme. Finally, we end the paper drawing some conclusions in Section 7.

\section{The eigenvalue problem}

We consider a coupled system composed of $K$ elastically mounted parallel tubes immersed in a fluid inside a rigid cylindrical cavity. Our problem is to determine the vibration modes of the system.

Under reasonable assumptions (see [5]), the problem can be posed in a two-dimensional framework, a planar transverse section of the cylindrical cavity being its domain. Each tube is modeled as a harmonic oscillator with rigidity $k$ and mass $m$ and the fluid is taken as perfectly incompressible with density $\rho$.

Let $\Omega \subset \mathbb{R}^{2}$ be the bounded domain occupied by the fluid, which we assume polygonal. Let $\Gamma_{0}$ be its outer boundary and $\Gamma_{i}, i=1, \ldots, K$, the interfaces between each tube and the fluid. Let $\boldsymbol{n}$ be the unit outer normal to the boundary of $\Omega$. (See Figure 1.)

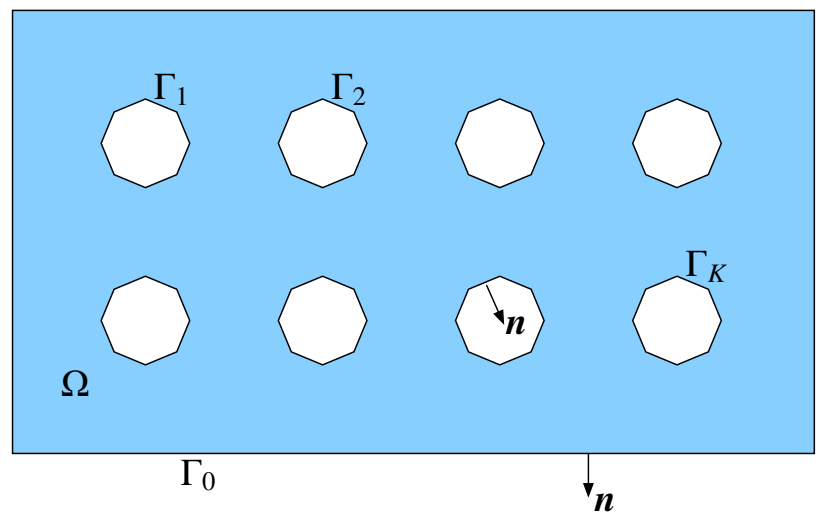

Figure 1: Sketch of the two-dimensional domain

The corresponding eigenvalue problem is the following one, which is known as the Laplace Model for fluid-solid vibrations $[4,5,8]$ :

Find $\omega>0$ (the vibration frequency) and $u \neq 0$ (the fluid pressure) such that

$$
\begin{cases}\Delta u=0 & \text { in } \Omega, \\ \frac{\partial u}{\partial n}=0 & \text { on } \Gamma_{0}, \\ \frac{\partial u}{\partial n}=\frac{\rho \omega^{2}}{k-m \omega^{2}}\left(\int_{\Gamma_{i}} u n\right) \cdot \boldsymbol{n} & \text { on } \Gamma_{i}, \quad i=1, \ldots, K .\end{cases}
$$

Let $\lambda:=\rho \omega^{2} /\left(k-m \omega^{2}\right)$ and $\mathscr{V}:=H^{1}(\Omega) / \mathbb{R}$ endowed with the $H^{1}$-seminorm, which is a norm on $\mathscr{V}$. The variational problem associated with (1) reads as follows:
Find $\lambda$ and $u \in \mathscr{V}$ satisfying

$$
\left\{\begin{array}{l}
a(u, v)=\lambda b(u, v) \quad \forall v \in \mathscr{V}, \\
b(u, u)=1
\end{array}\right.
$$

where

$$
a(u, v):=\int_{\Omega} \nabla u \cdot \nabla v \quad \text { and } \quad b(u, v):=\sum_{i=1}^{K}\left(\int_{\Gamma_{i}} u \boldsymbol{n}\right) \cdot\left(\int_{\Gamma_{i}} v \boldsymbol{n}\right)
$$

are continuous symmetric bilinear forms on $\mathscr{V}$, elliptic the former and non-negative the latter (i.e., $b(v, v) \geq 0 \forall v \in \mathscr{V}$ ).

The solution to (2) is given by a sequence of exactly $2 K$ pairs $\left(\lambda_{j}, u_{j}\right)$, with positive eigenvalues that we assume to be increasingly ordered: $0<\lambda_{1} \leq \cdots \leq \lambda_{2 K}$ (see [5, Section II.2.1]). Associated to each one there is an eigenfunction $u_{j} \in \mathscr{V}$, such that $\left\{u_{1}, \ldots, u_{2 K}\right\}$ is a linearly independent set.

\section{Finite element approximation and a-priori error esti- mates}

In this section we introduce an $h p$ finite element method for the spectral problem described in the previous section, prove its convergence and obtain a-priori error estimates for the eigenfunctions and the eigenvalues.

Let $\left\{\mathscr{T}_{h}\right\}$ be a family of triangulations of $\Omega$ such that any two triangles in $\mathscr{T}_{h}$ share at most a vertex or an edge. Let $h$ stand for the mesh-size; namely, $h:=\max _{T \in \mathscr{T}_{h}} h_{T}$, with $h_{T}$ being the diameter of the triangle $T$. We assume that the family of triangulations $\left\{\mathscr{T}_{h}\right\}$ satisfies a minimum angle condition and, consequently, there exists a constant $\sigma>0$ such that $h_{T} / r_{T} \leq$ $\sigma$, where $r_{T}$ is the diameter of the largest circle contained in $T$.

We associate with each element $T \in \mathscr{T}_{h}$ a (maximal) polynomial degree $p_{T} \in \mathbb{N}$. We assume that the polynomial degrees of neighboring elements are comparable, i.e., there exists a constant $\gamma>0$ such that

$$
\gamma^{-1} p_{T} \leq p_{T^{\prime}} \leq \gamma p_{T} \quad \forall T, T^{\prime} \in \mathscr{T}_{h} \text { with } T \cap T^{\prime} \neq \emptyset .
$$

We denote $\boldsymbol{p}:=\left\{p_{T}\right\}_{T \in \mathscr{T}_{h}}$, the family of polynomial degrees.

Throughout the paper, we will denote by $C$ a generic positive constant, not necessarily the same at each occurrence, which may depend on the mesh and the degree of the polynomials, only through the parameters $\sigma$ and $\gamma$, respectively.

We define the finite element space as follows:

$$
\mathscr{V}_{h}^{p}:=\left\{v \in \mathscr{V}: v_{T} \in \mathscr{P}_{p_{T}} \forall T \in \mathscr{T}_{h}\right\},
$$

where $\mathscr{P}_{k}$ denotes the space of polynomials of degree at most $k$. Notice that the definition of $\mathscr{V}_{h}^{p}$ allows for different maximal polynomial degrees on each edge of any triangle. Therefore, the space $\left\{\left.v\right|_{T}: v \in \mathscr{V}_{h}^{\boldsymbol{p}}\right\}$ does not necessarily coincides with $\mathscr{P}_{p_{T}}$. However, there exists $p_{T}^{\prime} \leq p_{T}$ such that

$$
\mathscr{P}_{p_{T}^{\prime}} \subset\left\{\left.v\right|_{T}: v \in \mathscr{V}_{h}^{p}\right\} \subset \mathscr{P}_{p_{T}}
$$

and $p_{T} / p_{T}^{\prime} \leq \gamma$ because of assumption (3).

The discrete eigenvalue problem associated with (2) is the following: 
Find $\lambda_{h}$ and $u_{h} \in \mathscr{V}_{h}^{\boldsymbol{p}}$ satisfying

$$
\left\{\begin{array}{l}
a\left(u_{h}, v_{h}\right)=\lambda_{h} b\left(u_{h}, v_{h}\right) \quad \forall v_{h} \in \mathscr{V}_{h}^{p}, \\
b\left(u_{h}, u_{h}\right)=1 .
\end{array}\right.
$$

This problem reduces to a generalized matrix eigenvalue problem. The theory in [5, Section II.2.1] holds also in this case and allows proving that this discrete problem attains $2 K$ positive eigenvalues, which we assume increasingly ordered: $0<\lambda_{h 1} \leq \cdots \leq \lambda_{h 2 K}$. Associated with each one, there is an eigenfunction $u_{h j} \in \mathscr{V}_{h}^{p}$, such that $\left\{u_{h 1}, \ldots, u_{h 2 K}\right\}$ is a linearly independent set.

Our first goal is to prove that the solutions of the discrete eigenvalue problem (5) converge to those of of the spectral problem (2). To do this, we will apply the classical spectral approximation theory from [24]. With this purpose, we introduce the bounded linear operators $T, T_{h}^{p}: \mathscr{V} \rightarrow \mathscr{V}$ defined by

$$
\begin{aligned}
& \left\{\begin{array}{l}
f \in \mathscr{V} \longmapsto T f \in \mathscr{V}, \\
a(T f, v)=b(f, v) \quad \forall v \in \mathscr{V},
\end{array}\right. \\
& \left\{\begin{array}{l}
f \in \mathscr{V} \longmapsto T_{h}^{\boldsymbol{p}} f \in \mathscr{V}_{h}^{\boldsymbol{p}} \subset \mathscr{V}, \\
a\left(T_{h}^{\boldsymbol{p}} f, v_{h}\right)=b\left(f, v_{h}\right) \quad \forall v_{h} \in \mathscr{V}_{h}^{\boldsymbol{p}} .
\end{array}\right.
\end{aligned}
$$

The non-zero eigenvalues of $T$ and $T_{h}^{p}$ are the reciprocals of the eigenvalues of (2) and (5), respectively.

On the other hand, from the standard a priori estimate for the Neumann problem (see [25]), we know that the solution to problem (6) satisfies

$$
T f \in H^{1+r}(\Omega)
$$

for all $r<\frac{\pi}{\theta}$, where $\theta$ is the largest reentrant angle of $\Omega$ and

$$
\|T f\|_{H^{1+r}(\Omega) / \mathbb{R}} \leq C|f|_{H^{1}(\Omega)} .
$$

Consequently, the eigenfunctions of problem (2) also satisfy $u \in H^{1+r}(\Omega)$ for all $r<\frac{\pi}{\theta}$. Let us remark that for a polygonal domain $\Omega$ with at least one interface $\Gamma_{i}$, necessarily $\theta>\pi$ and consequently $\frac{1}{2}<\frac{\pi}{\theta}<1$.

Let $p:=\min p$. The following proposition implies the convergence of $T_{h}^{p}$ to $T$ in norm as $h$ goes to 0 or $p$ goes to $\infty$.

Lemma 3.1. For $r<\frac{\pi}{\theta}$, there exists a positive constant $C$ such that, for all $f \in \mathscr{V}$,

$$
\left|T f-T_{h}^{p} f\right|_{H^{1}(\Omega)} \leq C\left(\frac{h}{p}\right)^{r}|f|_{H^{1}(\Omega)} .
$$

Proof. Since $a$ is $\mathscr{V}$-elliptic and $\mathscr{V}_{h}^{\boldsymbol{p}} \subset \mathscr{V}$, Cea's Lemma implies that, for all $f \in \mathscr{V}$,

$$
\left|T f-T_{h}^{p} f\right|_{H^{1}(\Omega)} \leq\left|T f-\Pi_{h}^{p}(T f)\right|_{H^{1}(\Omega)},
$$

where $\Pi_{h}^{p}$ denotes the $\mathscr{V}_{h}^{p}$-Lagrange interpolation operator (which is well defined because $T f \in H^{1+r}(\Omega)$, with $r>1 / 2$ ).

In its turn, by using standard $h p$ error estimates (see [26]) we obtain

$$
\begin{aligned}
\left|T f-\Pi_{h}^{p}(T f)\right|_{H^{1}(\Omega)}^{2} & \leq C \sum_{T \in \mathscr{T}_{h}}\left(\frac{h_{T}}{p_{T}^{\prime}}\right)^{2 r}\|T f\|_{H^{1+r}(T) / \mathbb{R}}^{2} \\
& \leq C\left(\frac{h}{p}\right)^{2 r}\|T f\|_{H^{1+r}(\Omega) / \mathbb{R}}^{2},
\end{aligned}
$$

where $p_{T}^{\prime}$ is as defined in (4). Thus, the result follows from (9).

As a consequence of the classical spectral approximation theory (see [24]) the eigenvalues and eigenfunctions of problem (5) converge to those of problem (2) as $h \rightarrow 0$ or $p \rightarrow \infty$. From now on, for simplicity, we restrict our attention to a simple eigenvalue $\lambda_{j}$ of problem (2) with corresponding eigenfunction $u_{j}$. Then, the $j$-th eigenvalue of problem (5), $\lambda_{h j}$, converges to $\lambda_{j}$ and the corresponding eigenfunction $u_{h j}$ can be chosen so that $u_{h j}$ converges to $u_{j}$, too.

In what follows we will adapt the techniques introduced in [27, Section 6.4] to obtain a priori error estimates for the approximate eigenvalues and eigenfunctions. Let us remark that we cannot use directly the results from this reference because the bilinear form $b$ is not an inner product in our case.

Since the number of eigenvalues is finite $(2 K)$, the convergence of the discrete eigenvalues stated above immediately implies that there exist $h_{0}>0$ and $p_{0} \in \mathbb{N}$ such that, if $h \leq h_{0}$ or $p \geq p_{0}$, then $\lambda_{h i} \neq \lambda_{j}$ for all $i \neq j, 1 \leq i \leq 2 K$. In such a case, we are allowed to define

$$
\rho_{h j}:=\max _{\substack{1 \leq i \leq 2 K \\ i \neq j}} \frac{\lambda_{j}}{\left|\lambda_{h i}-\lambda_{j}\right|} .
$$

Let $P_{h}^{p}$ denote the $\mathscr{V}$-elliptic projection onto $\mathscr{V}_{h}^{p}$ defined for any $w \in \mathscr{V}$ by

$$
P_{h}^{p} w \in \mathscr{V}_{h}^{\boldsymbol{p}}: \quad a\left(P_{h}^{\boldsymbol{p}} w-w, v_{h}\right)=0 \quad \forall v_{h} \in \mathscr{V}_{h}^{\boldsymbol{p}} .
$$

The following lemma extends to our problem the results from [27, Lemma 6.4-3].

Lemma 3.2. There exist $h_{0}>0$ and $p_{0} \in \mathbb{N}$ such that, if $h \leq h_{0}$ or $p \geq p_{0}$, then the discrete eigenfunction $u_{h j}$ can be chosen so that

$b\left(u_{j}-u_{h j}, u_{j}-u_{h j}\right)^{1 / 2} \leq 2\left(1+\rho_{h j}\right) b\left(u_{j}-P_{h}^{p} u_{j}, u_{j}-P_{h}^{p} u_{j}\right)^{1 / 2}$.

Proof. We do not include the whole proof, since it follows closely the arguments used in [27] to prove Lemma 6.4-3. The main difference is that, in our case, the set of discrete eigenfunctions $\left\{u_{h 1}, \ldots, u_{h 2 K}\right\}$ is not a basis of $\mathscr{V}_{h}^{\boldsymbol{p}}$. However, this linearly independent set can be chosen such that $b\left(u_{h i}, u_{h j}\right)=\delta_{i j}$, $1 \leq i, j \leq 2 K$, and it can be completed to a basis $\left\{u_{h 1}, \ldots, u_{h N}\right\}$ $\left(N:=\operatorname{dim} \mathscr{V}_{h}^{\boldsymbol{p}}\right)$, with the added basis functions satisfying

$$
b\left(u_{h j}, v_{h}\right)=0 \quad \forall v_{h} \in \mathscr{V}_{h}^{p}, \quad j=2 K+1, \ldots, N .
$$

Therefore, $b\left(u_{h i}, u_{h j}\right)=0$ if $i \neq j, 1 \leq i, j \leq N$. Using this basis, the proof of Lemma 6.4-3 from [27] can be conveniently adapted to obtain (11).

Now we are in a position to prove the following a priori error estimates. 
Proposition 3.1. For all $r<\frac{\pi}{\theta}$, there exist positive constants $C$ and $\kappa$, such that, if $\frac{h}{p}<\kappa$, then

$$
\begin{aligned}
\left|u_{j}-u_{h j}\right|_{H^{1}(\Omega)} & \leq C\left(\frac{h}{p}\right)^{r}, \\
b\left(u_{j}-u_{h j}, u_{j}-u_{h j}\right)^{1 / 2} & \leq C\left(\frac{h}{p}\right)^{r}\left|u_{j}-u_{h j}\right|_{H^{1}(\Omega)}, \\
\left|\lambda_{j}-\lambda_{h j}\right| & \leq C\left|u_{j}-u_{h j}\right|_{H^{1}(\Omega)}^{2} .
\end{aligned}
$$

Proof. The estimate (12) is a direct consequence of Lemma 3.1 and the classical spectral approximation theory (see [24]).

To prove (13), we use Lemma 3.2 and a duality argument to estimate the right-hand side of (11). Let $\varphi \in \mathscr{V}$ be the solution to

$$
a(\psi, \varphi)=b\left(\psi, u_{j}-P_{h}^{p} u_{j}\right) \quad \forall \psi \in \mathscr{V} .
$$

Hence, $\varphi$ satisfies

$$
\begin{cases}\Delta \varphi=0 & \text { in } \Omega, \\ \frac{\partial \varphi}{\partial n}=0 & \text { on } \Gamma_{0}, \\ \frac{\partial \varphi}{\partial n}=c_{i} \cdot n & \text { on } \Gamma_{i}, \quad i=1, \ldots, K,\end{cases}
$$

with $c_{i}:=\int_{\Gamma_{i}}\left(u_{j}-P_{h}^{p} u_{j}\right) \boldsymbol{n}$. The same arguments leading to (8)-(9) allow us to conclude that $\varphi \in H^{1+r}(\Omega)$ and

$\|\varphi\|_{H^{1+r}(\Omega) / \mathbb{R}} \leq C\left(\sum_{i=1}^{K}\left|c_{i}\right|^{2}\left|\Gamma_{i}\right|\right)^{1 / 2} \leq C b\left(u_{j}-P_{h}^{p} u_{j}, u_{j}-P_{h}^{p} u_{j}\right)^{1 / 2}$,

where, for the first inequality, we have used on each straight segment $\gamma$ of $\Gamma_{i}$ that $\left\|\boldsymbol{c}_{i} \cdot \boldsymbol{n}\right\|_{H^{1 / 2}(\gamma)}^{2}=\left\|\boldsymbol{c}_{i} \cdot \boldsymbol{n}\right\|_{L^{2}(\gamma)}^{2} \leq\left|\boldsymbol{c}_{i}\right|^{2}|\gamma|$, because $\boldsymbol{c}_{i} \cdot \boldsymbol{n}$ is constant on $\gamma$.

Taking $\psi=u_{j}-P_{h}^{p} u_{j}$ in (15) and using (10), we obtain

$$
\begin{aligned}
b\left(u_{j}-P_{h}^{p} u_{j}, u_{j}-P_{h}^{p} u_{j}\right) & =a\left(u_{j}-P_{h}^{p} u_{j}, \varphi\right) \\
& =a\left(u_{j}-P_{h}^{p} u_{j}, \varphi-\Pi_{h}^{p} \varphi\right) \\
& \leq\left|u_{j}-P_{h}^{p} u_{j}\right|_{H^{1}(\Omega)}\left|\varphi-\Pi_{h}^{p} \varphi\right|_{H^{1}(\Omega)},
\end{aligned}
$$

where, once more, $\Pi_{h}^{p}$ denotes the $\mathscr{V}_{h}^{p}$-Lagrange interpolation operator. Now, using again standard $h p$ error estimates (see [26]), we have

$$
\left|\varphi-\Pi_{h}^{p} \varphi\right|_{H^{1}(\Omega)} \leq C\left(\frac{h}{p}\right)^{r}\|\varphi\|_{H^{1+r}(\Omega) / \mathbb{R}} .
$$

Then, (13) follows from (11), (17), (18) and (16), together with the inequality $\left|u_{j}-P_{h}^{p}\left(u_{j}\right)\right|_{H^{1}(\Omega)} \leq\left|u_{j}-u_{h j}\right|_{H^{1}(\Omega)}$, which holds because $P_{h}^{\boldsymbol{p}}$ is the projector onto $\mathscr{V}_{h}^{\boldsymbol{p}}$.

Finally (14) follows from (12), (13) and the well known identity (see, for instance, Lemma 9.1 from [24])

$$
\lambda_{h j}-\lambda_{j}=a\left(u_{h j}-u_{j}, u_{h j}-u_{j}\right)-\lambda_{j} b\left(u_{h j}-u_{j}, u_{h j}-u_{j}\right) .
$$

Thus we conclude the proof.

\section{A posteriori error estimator}

In this section we introduce an a posteriori estimator for the error in the energy norm of the approximate eigenfunction and prove its reliability and efficiency. From now on we drop the subindex $j$ in $\lambda_{j}, \lambda_{h j}, u_{j}$ and $u_{h j}$.

We introduce some notation that we will use in the definition and analysis of the error estimator. For any $T \in \mathscr{T}_{h}$ let $\mathscr{E}_{T}$ denote the set of edges of $T$ and $\mathscr{E}:=\bigcup_{T \in \mathscr{T}_{h}} \mathscr{E}_{T}$. We decompose $\mathscr{E}$ in disjoint sets $\mathscr{E}_{\Gamma_{i}}:=\left\{\ell \in \mathscr{E}: \ell \subset \Gamma_{i}\right\}, 0 \leq i \leq K$, and $\mathscr{E}_{\Omega}:=\mathscr{E} \backslash \bigcup_{i=0}^{K} \mathscr{E}_{\Gamma_{i}}$

For each $\ell \in \mathscr{E}_{\Omega}$ we choose a unit normal vector $\boldsymbol{n}_{\ell}$ and denote the two triangles sharing this edge $T_{\text {in }}$ and $T_{\text {out }}$, with $\boldsymbol{n}_{\ell}$ pointing outwards $T_{\text {in }}$. For $v_{h} \in \mathscr{V}_{h}$ we set

$$
\llbracket \frac{\partial v_{h}}{\partial n} \|_{\ell}:=\nabla\left(\left.v_{h}\right|_{T_{\text {out }}}\right) \cdot \boldsymbol{n}_{\ell}-\nabla\left(\left.v_{h}\right|_{T_{\text {in }}}\right) \cdot \boldsymbol{n}_{\ell},
$$

which corresponds to the jump of the normal derivative of $v_{h}$ across the edge $\ell$. Notice that this value is independent of the chosen direction of the normal vector $\boldsymbol{n}_{\ell}$.

From (2) and (5), we know that for any $v_{h} \in \mathscr{V}_{h}^{\boldsymbol{p}}$ the error $e:=u-u_{h}$ satisfies

$$
\int_{\Omega} \nabla e \cdot \nabla v_{h}=\sum_{i=1}^{K}\left(\lambda \int_{\Gamma_{i}} u \boldsymbol{n}-\lambda_{h} \int_{\Gamma_{i}} u_{h} \boldsymbol{n}\right) \cdot\left(\int_{\Gamma_{i}} v_{h} \boldsymbol{n}\right)
$$

On the other hand, for any $v \in \mathscr{V}$, using (2) and integrating by parts we obtain

$$
\begin{aligned}
\int_{\Omega} \nabla e \cdot \nabla v= & \sum_{i=1}^{K} \lambda\left(\int_{\Gamma_{i}} u \boldsymbol{n}\right) \cdot\left(\int_{\Gamma_{i}} v \boldsymbol{n}\right) \\
& +\sum_{T \in \mathscr{T}_{h}} \int_{T} \Delta u_{h} v+\sum_{T \in \mathscr{T}_{h}} \int_{\partial T} \frac{\partial u_{h}}{\partial n} v .
\end{aligned}
$$

Hence, defining for each edge $\ell \in \mathscr{E}$

$$
J_{\ell}:= \begin{cases}\frac{1}{2} \llbracket \frac{\partial u_{h}}{\partial n} \|_{\ell}, & \ell \in \mathscr{E}_{\Omega}, \\ \frac{\partial u_{h}}{\partial n}, & \ell \in \mathscr{E}_{\Gamma_{0}}, \\ \frac{\partial u_{h}}{\partial n}-\left(\int_{\Gamma_{i}} \lambda_{h} u_{h} n\right) \cdot \boldsymbol{n}, & \ell \in \mathscr{E}_{\Gamma_{i}}, \quad i=1, \ldots, K,\end{cases}
$$

straightforward computations allow us to write, for all $v \in \mathscr{V}$,

$$
\begin{aligned}
\int_{\Omega} \nabla e \cdot \nabla v= & \sum_{T \in \mathscr{T}_{h}}\left(\int_{T} \Delta u_{h} v+\sum_{\ell \in \mathscr{E}_{T}} \int_{\ell} J_{\ell} v\right) \\
& +\sum_{i=1}^{K}\left(\lambda \int_{\Gamma_{i}} u \boldsymbol{n}-\lambda_{h} \int_{\Gamma_{i}} u_{h} \boldsymbol{n}\right) \cdot\left(\int_{\Gamma_{i}} v \boldsymbol{n}\right) .
\end{aligned}
$$

For each element $T \in \mathscr{T}_{h}$, we define the local error indicator $\eta_{T}$ by

$$
\eta_{T}^{2}:=\frac{h_{T}^{2}}{p_{T}^{2}}\left\|\Delta u_{h}\right\|_{L^{2}(T)}^{2}+\sum_{\ell \in \mathscr{E}_{T}} \frac{|\ell|}{p_{\ell}}\left\|J_{\ell}\right\|_{L^{2}(\ell)}^{2}
$$


with $p_{\ell}:=\max \left\{p_{T}: \ell \in \mathscr{E}_{T}\right\}$, and the global error estimator $\eta_{\Omega}$ by

$$
\eta_{\Omega}^{2}:=\sum_{T \in \mathscr{T}_{h}} \eta_{T}^{2}
$$

To compare the error and the estimator we will use an $h p$ Clément interpolation operator $I_{h}^{p}: \mathscr{V} \rightarrow \mathscr{V}_{h}^{\boldsymbol{p}}$ defined in [16]. In this reference it is shown that this operator satisfies the following error estimates:

$$
\begin{array}{ll}
\left\|u-I_{h}^{p} u\right\|_{L^{2}(T)} \leq C \frac{h_{V}}{p_{V}}|u|_{H^{1}\left(\omega_{V}^{4}\right)} & \forall T \in \mathscr{T}_{h}: T \subset \omega_{V}^{1}, \\
\left\|u-I_{h}^{p} u\right\|_{L^{2}(\ell)} \leq C\left(\frac{h_{V}}{p_{V}}\right)^{\frac{1}{2}}|u|_{H^{1}\left(\omega_{V}^{4}\right)} & \forall \ell \in \mathscr{E}_{V},
\end{array}
$$

where, for each vertex $V$ of the triangulation $\mathscr{T}_{h}$,

$$
\begin{aligned}
\omega_{V}^{0} & :=\{V\}, \\
\omega_{V}^{j} & :=\bigcup\left\{T \in \mathscr{T}_{h}: T \cap \omega_{V}^{j-1} \neq \emptyset\right\}, \quad j \geq 1, \\
h_{V} & :=\max \left\{h_{T}: V \text { is a vertex of } T\right\}, \\
p_{V} & :=\max \left\{p_{T}+1: V \text { is a vertex of } T\right\}, \\
\mathscr{E}_{V} & :=\{\ell \in \mathscr{E}: V \text { is an endpoint of } \ell\} .
\end{aligned}
$$

We observe in the estimates above that the subdomain $\omega_{V}^{4}$ is larger than the one appearing in the error estimates for the classical $h$-Clément interpolant. However this will only affect the size of the constants in the estimates.

The following theorem provides an upper bound for the error, which proves the reliability of the error estimator up to higher order terms.

Theorem 4.1. There exists a positive constant $C$ such that

$$
|e|_{H^{1}(\Omega)} \leq C\left[\eta_{\Omega}+\left(\frac{h}{p}\right)^{2 r}|e|_{H^{1}(\Omega)}\right] .
$$

Proof. By using the error equations (19) with $v_{h}=I_{h}^{p} e$ and (20) with $v=e-I_{h}^{p} e$, we obtain

$$
\begin{aligned}
|e|_{H^{1}(\Omega)}^{2}= & \int_{\Omega} \nabla e \cdot \nabla\left(e-I_{h}^{p} e\right)+\int_{\Omega} \nabla e \cdot \nabla\left(I_{h}^{p} e\right) \\
= & \sum_{T \in \mathscr{T}_{h}}\left[\int_{T} \Delta u_{h}\left(e-I_{h}^{p} e\right)+\sum_{\ell \in \mathscr{E}_{T}} \int_{\ell} J_{\ell}\left(e-I_{h}^{p} e\right)\right] \\
& +\sum_{i=1}^{K}\left(\lambda \int_{\Gamma_{i}} u \boldsymbol{n}-\lambda_{h} \int_{\Gamma_{i}} u_{h} \boldsymbol{n}\right) \cdot\left(\int_{\Gamma_{i}} e \boldsymbol{n}\right) .
\end{aligned}
$$

Next, we estimate separately the two terms on the right hand side above.

For each $T \in \mathscr{T}_{h}$, let $V_{T}$ be one of the vertices of $T$ and for each edge $\ell \in \mathscr{E}$ let $V_{\ell}$ be one of the endpoints of $\ell$. Then, by using the Cauchy-Schwartz inequality, (22), (23), the definition of $\eta_{\Omega}$ and the fact that the triangulation satisfies the minimum angle condition and (3), we obtain

$$
\begin{aligned}
\sum_{T \in \mathscr{T}_{h}}\left[\int_{T} \Delta u_{h}\left(e-I_{h}^{p} e\right)+\sum_{\ell \in \mathscr{E}_{T}} \int_{\ell} J_{\ell}\left(e-I_{h}^{p} e\right)\right] \\
\leq C \sum_{T \in \mathscr{T}_{h}}\left[\frac{h_{V_{T}}}{p_{V_{T}}}\left\|\Delta u_{h}\right\|_{L^{2}(T)}|e|_{H^{1}\left(\omega_{V_{T}}^{4}\right)}\right. \\
\left.+\sum_{\ell \in \mathscr{E}_{T}}\left(\frac{h_{V_{\ell}}}{p_{V_{\ell}}}\right)^{\frac{1}{2}}\left\|J_{\ell}\right\|_{L^{2}(\ell)}|e|_{H^{1}\left(\omega_{V_{\ell}}^{4}\right)}\right]
\end{aligned}
$$$$
\leq C \eta_{\Omega}|e|_{H^{1}(\Omega)} \text {. }
$$

On the other hand, since $b(u, u)=1$ and $b\left(u_{h}, u_{h}\right)=1$, we have

$$
\begin{aligned}
\sum_{i=1}^{K}\left(\lambda \int_{\Gamma_{i}}\right. & \left.u \boldsymbol{n}-\lambda_{h} \int_{\Gamma_{i}} u_{h} \boldsymbol{n}\right) \cdot\left(\int_{\Gamma_{i}} e \boldsymbol{n}\right) \\
= & \lambda b(u, u)-\left(\lambda+\lambda_{h}\right) b\left(u, u_{h}\right)+\lambda_{h} b\left(u_{h}, u_{h}\right) \\
& =\left(\lambda+\lambda_{h}\right)-\left(\lambda+\lambda_{h}\right) b\left(u, u_{h}\right) \\
& =\frac{\lambda+\lambda_{h}}{2} b\left(u-u_{h}, u-u_{h}\right) \\
& \leq C\left(\frac{h}{p}\right)^{2 r}|e|_{H^{1}(\Omega)}^{2},
\end{aligned}
$$

where we have used (13) for the last inequality. Thus we conclude the proof.

In order to guarantee that the error indicator is efficient to guide an adaptive refinement scheme, our next goal is to prove that $\eta_{T}$ is bounded by the $H^{1}$ norm of the error on a neighborhood of $T$, up to higher order terms.

For $T \in \mathscr{T}_{h}$, let $b_{T}$ be the standard cubic bubble given by

$$
b_{T}:= \begin{cases}\lambda_{1}^{T} \lambda_{2}^{T} \lambda_{3}^{T}, & \text { in } T, \\ 0, & \text { in } \Omega \backslash T,\end{cases}
$$

where $\lambda_{1}^{T}, \lambda_{2}^{T}$ and $\lambda_{3}^{T}$ denote the barycentric coordinates of $T$.

For $\ell \in \mathscr{E}_{\Omega}$, we denote by $T_{1}$ and $T_{2}$ the two triangles sharing $\ell$ and we enumerate the vertices of $T_{1}$ and $T_{2}$ so that the vertices of $\ell$ are numbered first. Then we consider the piecewise quadratic edge bubble function $b_{\ell}$ defined by

$$
b_{\ell}:= \begin{cases}\lambda_{1}^{T_{i}} \lambda_{2}^{T_{i}}, & \text { in } T_{i}, \quad i=1,2, \\ 0, & \text { in } \Omega \backslash T_{1} \cup T_{2} .\end{cases}
$$

The following lemma provides an upper estimate for the first term in the definition of $\eta_{T}$ (cf. (21)).

Lemma 4.1. There exists a positive constant $C$ such that

$$
\frac{h_{T}}{p_{T}}\left\|\Delta u_{h}\right\|_{L^{2}(T)} \leq C p_{T}|e|_{H^{1}(T)} .
$$

Proof. Using (20) with $v=\Delta u_{h} b_{T} \in H_{0}^{1}(T) \subset \mathscr{V}$, we obtain

$$
\begin{aligned}
\int_{T}\left(\Delta u_{h}\right)^{2} b_{T} & =\int_{T} \nabla e \cdot \nabla\left(\Delta u_{h} b_{T}\right) \\
& \leq C \frac{p_{T}}{h_{T}}|e|_{H^{1}(T)}\left[\int_{T}\left(\Delta u_{h}\right)^{2} b_{T}\right]^{1 / 2},
\end{aligned}
$$


where we have applied to $\Delta u_{h} b_{T} \in \mathscr{P}_{p_{T}+1}$, an inverse inequality proved in [16] (see equation (24) from this reference). Hence, using equation (22) from [16], we obtain

$$
\left\|\Delta u_{h}\right\|_{L^{2}(T)} \leq C p_{T}\left[\int_{T}\left(\Delta u_{h}\right)^{2} b_{T}\right]^{1 / 2} \leq C \frac{p_{T}^{2}}{h_{T}}|e|_{H^{1}(T)},
$$

from which we conclude the proof.

Next, we prove an upper estimate for the second term in the definition of $\eta_{T}$ (cf. (21)).

Lemma 4.2. For all $\delta>0$, there exists a positive constant $C_{\delta}$ such that, if $\ell \in \mathscr{E}_{\Omega} \cup \mathscr{E}_{\Gamma_{0}}$, then

$$
\frac{|\ell|^{1 / 2}}{p_{\ell}^{1 / 2}}\left\|J_{\ell}\right\|_{L^{2}(\ell)} \leq C_{\delta} p_{\ell}^{1+\delta}|e|_{H^{1}\left(\omega_{\ell}\right)}
$$

and, if $\ell \in \mathscr{E}_{\Gamma_{i}}, 1 \leq i \leq K$, then

$$
\frac{|\ell|^{1 / 2}}{p_{\ell}^{1 / 2}}\left\|J_{\ell}\right\|_{L^{2}(\ell)} \leq C_{\delta}\left[p_{\ell}^{1+\delta}|e|_{H^{1}\left(\omega_{\ell}\right)}+p_{\ell}^{\delta}|\ell|\left|\int_{\Gamma_{i}}\left(\lambda u-\lambda_{h} u_{h}\right) n\right|\right],
$$

where $\omega_{\ell}:=\bigcup\left\{T \in \mathscr{T}_{h}: \ell \in \mathscr{E}_{T}\right\}$.

Proof. We follow the arguments proposed in [16]. According to Lemma 2.4 from this reference, for all $\beta>0$ there exists $C_{\beta}>0$, only depending on $\beta$, such that

$$
\left\|J_{\ell}\right\|_{L^{2}(\ell)} \leq C_{\beta} p_{\ell}^{\beta}\left(\int_{\ell} b_{\ell}^{\beta} J_{\ell}^{2}\right)^{1 / 2},
$$

Moreover, using Lemma 2.6 from the same reference ([16]) and standard scaling arguments, we have that for all $\beta>\frac{1}{2}$, there exists another constant $C_{\beta}>0$, again depending only on $\beta$, such that for all $\epsilon>0$, there exists $v_{\epsilon} \in H_{0}^{1}\left(\omega_{\ell}\right)$ satisfying

$$
\begin{aligned}
\left.v_{\epsilon}\right|_{\ell} & =b_{\ell}^{\beta} J_{\ell} \\
\left\|v_{\epsilon}\right\|_{L^{2}\left(\omega_{\ell}\right)}^{2} & \leq C_{\beta} \epsilon|\ell| \int_{\ell} b_{\ell}^{\beta} J_{\ell}^{2}, \\
\left|v_{\epsilon}\right|_{H^{1}\left(\omega_{\ell}\right)}^{2} & \leq C_{\beta}\left[\epsilon p_{\ell}^{2(2-\beta)}+\epsilon^{-1}\right] \frac{1}{|\ell|} \int_{\ell} b_{\ell}^{\beta} J_{\ell}^{2} .
\end{aligned}
$$

For $\ell \in \mathscr{E}_{\Omega} \cup \mathscr{E}_{\Gamma_{0}}$, we use (20) with $v=v_{\epsilon}$ to write

$$
\int_{\omega_{\ell}} \nabla e \cdot \nabla v_{\epsilon}=\int_{\omega_{\ell}} \Delta u_{h} v_{\epsilon}+\int_{\ell} J_{\ell} v_{\epsilon}
$$

Hence, using (28), (30), (29) and (24), we obtain

$$
\begin{aligned}
\int_{\ell} b_{\ell}^{\beta} J_{\ell}^{2} & =\int_{\ell} J_{\ell} v_{\epsilon} \\
& \leq|e|_{H^{1}\left(\omega_{\ell}\right)}\left|v_{\epsilon}\right|_{H^{1}\left(\omega_{\ell}\right)}+\left\|\Delta u_{h}\right\|_{L^{2}\left(\omega_{\ell}\right)}\left\|v_{\epsilon}\right\|_{L^{2}\left(\omega_{\ell}\right)} \\
& \leq \frac{C_{\beta}}{|\ell|^{1 / 2}}\left[\epsilon p_{\ell}^{2(2-\beta)}+\epsilon^{-1}+\epsilon p_{\ell}^{4}\right]^{1 / 2}|e|_{H^{1}\left(\omega_{\ell}\right)}\left(\int_{\ell} b_{\ell}^{\beta} J_{\ell}^{2}\right)^{1 / 2} .
\end{aligned}
$$

Choosing $\epsilon=p_{\ell}^{-2}$ in this estimate, we have

$$
\left(\int_{\ell} b_{\ell}^{\beta} J_{\ell}^{2}\right)^{1 / 2} \leq C_{\beta} \frac{p_{\ell}}{|\ell|^{1 / 2}}|e|_{H^{1}\left(\omega_{\ell}\right)}
$$

from which, taking $\beta=\frac{1}{2}+\delta$ and using (27), we obtain (25).

Next, for $\ell \in \mathscr{E}_{\Gamma_{i}}, i=1, \ldots, K$, we use (20) with $v=v_{\epsilon}$ to write

$$
\begin{aligned}
\int_{\omega_{\ell}} \nabla e \cdot \nabla v_{\epsilon}= & \int_{\omega_{\ell}} \Delta u_{h} v_{\epsilon}+\int_{\ell} J_{\ell} v_{\epsilon} \\
& +\left(\int_{\Gamma_{i}} \lambda u \boldsymbol{n}-\int_{\Gamma_{i}} \lambda_{h} u_{h} \boldsymbol{n}\right) \cdot\left(\int_{\ell} v_{\epsilon} \boldsymbol{n}_{\ell}\right) .
\end{aligned}
$$

Proceeding as in the previous case, we obtain now

$$
\left(\int_{\ell} b_{\ell}^{\beta} J_{\ell}^{2}\right)^{1 / 2} \leq C_{\beta} \frac{p_{\ell}}{|\ell|^{1 / 2}}|e|_{H^{1}\left(\omega_{\ell}\right)}+|\ell|^{1 / 2}\left|\int_{\Gamma_{i}}\left(\lambda u-\lambda_{h} u_{h}\right) n\right|,
$$

from which (26) follows. Thus we conclude the proof.

Now we may conclude the efficiency of the error indicator.

Theorem 4.2. For all $\delta>0$, there exists a positive constant $C_{\delta}$ such that for all $T \in \mathscr{T}_{h}$, if $T$ has only inner edges (i.e., edges $\left.\ell \in \mathscr{E}_{\Omega}\right)$, then

$$
\eta_{T} \leq C_{\delta} p_{T}^{1+\delta}|e|_{H^{1}\left(\omega_{T}\right)}
$$

and, if $T$ has an edge lying on $\Gamma_{i}, i=1, \ldots, K$, then

$$
\eta_{T} \leq C_{\delta} p_{T}^{1+\delta}\left[|e|_{H^{1}\left(\omega_{T}\right)}+\frac{h_{T}}{p_{T}}\left|\int_{\Gamma_{i}}\left(\lambda u-\lambda_{h} u_{h}\right) n\right|\right],
$$

where $\omega_{T}:=\bigcup\left\{T^{\prime}: T\right.$ and $T^{\prime}$ share an edge $\}$.

Proof. It is an immediate consequence of Lemmas 4.1 and 4.2 and the assumption (3).

Notice that the efficiency estimate above is suboptimal in that the equivalence constant depends on the polynomial degree. In contrast to the case of $h$ refinement, it seems to be an open question whether uniform reliability and efficiency can be achieved for an $h p$ a posteriori estimator. In fact, to the best of the authors' knowledge, proofs of upper and lower bounds both independent of the polynomial degree $p$ have not been reported yet for any $h p$ finite element method. Nevertheless, according to the experiments reported in Section 6, this seems to be just a theoretical issue. Indeed, the degrees achieved in the experiments are not that large, so that the factor $p^{1+\delta}$ can be considered bounded for practical purposes.

Remark 4.1. From Lemmas 4.1 and 4.2, we also obtain for all $\delta>0$ the following global lower error estimate:

$$
\eta_{\Omega} \leq C_{\delta}(\max \boldsymbol{p})^{1+\delta}\left(|e|_{H^{1}(\Omega)}^{2}+\text { h.o.t. }\right)^{1 / 2}
$$

where

$$
\begin{aligned}
\text { h.o.t. } & :=\frac{1}{p^{2}} \sum_{i=1}^{K}\left[\sum_{\ell \in \mathscr{E}_{\Gamma_{i}}}|\ell|^{2}\left|\int_{\Gamma_{i}}\left(\lambda u-\lambda_{h} u_{h}\right) n\right|^{2}\right] \\
& \leq \frac{2 h}{p^{2}} \sum_{i=1}^{K}\left|\Gamma_{i}\right|\left[|\lambda|^{2}\left|\int_{\Gamma_{i}}\left(u-u_{h}\right) n\right|^{2}+\left|\lambda-\lambda_{h}\right|^{2}\left|\int_{\Gamma_{i}} u_{h} n\right|^{2}\right] .
\end{aligned}
$$

Thus, from (13), (14) and the fact that $b\left(u_{h}, u_{h}\right)=1$, we have

$$
\text { h.o.t. } \leq \frac{2 h}{p^{2}} \sum_{i=1}^{K}\left|\Gamma_{i}\right|\left[|\lambda|^{2}\left(\frac{h}{p}\right)^{2 r}|e|_{H^{1}(\Omega)}^{2}+|e|_{H^{1}(\Omega)}^{4}\right],
$$

which is clearly a higher order term as compared with $|e|_{H^{1}(\Omega)}^{2}$. 


\section{Numerical aspects}

In this section we analyze numerical aspects concerning the solution of the discrete problem (5) and introduce an adaptive refinement strategy based on the indicators $\eta_{T}$.

\subsection{Solution of the generalized eigenvalue problem}

The finite element space used for the discretization is $\mathscr{V}_{h}^{\boldsymbol{p}}=$ $\mathscr{W}_{h}^{p} / \mathbb{R}$ with

$$
\mathscr{W}_{h}^{p}:=\left\{v \in H^{1}(\Omega):\left.v\right|_{T} \in \mathscr{P}_{p_{T}} \forall T \in \mathscr{T}_{h}\right\}
$$

We denote by $\mathscr{N}$ the set of nodes of $\mathscr{W}_{h}^{p}$ and decompose this set as follows: $\mathscr{N}=\mathscr{N}_{1} \cup \mathscr{N}_{2}$, with $\mathscr{N}_{1}$ being the subset of nodes lying on $\bigcup_{i=1}^{K} \Gamma_{i}$ and $\mathscr{N}_{2}$ the subset of the remaining ones (i.e., those lying either in $\Omega$ or on $\Gamma_{0}$ ). We denote by $N_{i}$ the number of nodes on $\mathscr{N}_{i}, i=1,2$.

Let

$$
\boldsymbol{u}_{1}:=\left(u\left(P_{i}\right)\right)_{P_{i} \in \mathscr{N}_{1}} \in \mathbb{R}^{N_{1}} \quad \text { and } \quad \boldsymbol{u}_{2}:=\left(u\left(P_{i}\right)\right)_{P_{i} \in \mathscr{N}_{2}} \in \mathbb{R}^{N_{2}} .
$$

To obtain a matrix form of the discrete problem (5), we write

$$
\left(\begin{array}{ll}
\boldsymbol{A}_{11} & \boldsymbol{A}_{12} \\
\boldsymbol{A}_{21} & \boldsymbol{A}_{22}
\end{array}\right)\left(\begin{array}{l}
\boldsymbol{u}_{1} \\
\boldsymbol{u}_{2}
\end{array}\right)=\lambda_{h}\left(\begin{array}{cc}
\boldsymbol{B}_{11} & \mathbf{0} \\
\mathbf{0} & \mathbf{0}
\end{array}\right)\left(\begin{array}{l}
\boldsymbol{u}_{1} \\
\boldsymbol{u}_{2}
\end{array}\right)
$$

with

$$
\begin{aligned}
\boldsymbol{A}_{r s} & :=\left(a\left(\beta_{i}, \beta_{j}\right)\right)_{P_{i} \in \mathscr{N}_{r}, P_{j} \in \mathscr{N}_{s}}, \quad r, s=1,2, \\
\boldsymbol{B}_{11} & :=\left(b\left(\beta_{i}, \beta_{j}\right)\right)_{P_{i}, P_{j} \in \mathscr{N}_{1}},
\end{aligned}
$$

with $\left\{\beta_{i}\right\}_{P_{i} \in \mathscr{N}}$ being the nodal basis of $\mathscr{W}_{h}^{p}$ (i.e., $\beta_{i}\left(P_{j}\right)=\delta_{i j}$ ).

The matrices on the left and right hand sides of (31) are symmetric and positive semi-definite. However, this eigenvalue problem is degenerate because the kernels of both matrices contain the vector $(1, \ldots, 1)^{\mathrm{t}} \in \mathbb{R}^{N_{1}+N_{2}}$. In fact, problems (5) and (31) are not equivalent, since the former is posed on the quotient space $\mathscr{V}_{h}^{\boldsymbol{p}}=\mathscr{W}_{h}^{\boldsymbol{p}} / \mathbb{R}$ and the latter on $\mathscr{W}_{h}^{\boldsymbol{p}}$. To obtain a matrix form of (5), it is enough to set to zero one arbitrary component of $\boldsymbol{u}_{1}$ or $\boldsymbol{u}_{2}$. This is easily done by deleting in both matrices from (31) the row and the column corresponding to the nodal component set to zero. Then, the resulting generalized eigenvalue problem is well posed because the matrix on the left hand side is symmetric and positive definite.

However, with the aim of reducing the computational cost, we followed an alternative way. First, since the submatrix $\boldsymbol{A}_{22}$ is invertible (indeed, symmetric and positive definite), by eliminating $\boldsymbol{u}_{2}$ from (31) we arrive at

$$
\left(\boldsymbol{A}_{11}-\boldsymbol{A}_{12} \boldsymbol{A}_{22}^{-1} \boldsymbol{A}_{21}\right) \boldsymbol{u}_{1}=\lambda_{h} \boldsymbol{B}_{11} \boldsymbol{u}_{1},
$$

This problem is equivalent to (31). Moreover, both matrices are again symmetric and positive semi-definite, with a common non-zero vector $(1, \ldots, 1)^{\mathrm{t}} \in \mathbb{R}^{N_{1}}$ in their kernels. Thus, problem (32) is also degenerate.

Notice that although the matrix on the left hand side of (32) is not sparse, its size is $N_{1}$ and, hence, significantly smaller than the size $N_{1}+N_{2}$ of problem (31). Moreover, in actual computations, the matrix $\boldsymbol{A}_{22}^{-1}$ is not explicitly computed. In fact, the columns of $\boldsymbol{A}_{22}^{-1} \boldsymbol{A}_{12}$ are obtained by solving $N_{1}$ linear systems with the same matrix $\boldsymbol{A}_{22} \in \mathbb{R}^{N_{2} \times N_{2}}$, which is sparse, symmetric and positive definite.

As a second step, we compute a complete diagonalization of the matrix $\boldsymbol{B}_{11}$. As far as the mesh have no triangles with vertices lying on two different $\Gamma_{i}$, this matrix is block diagonal with $K$ full diagonal blocks, the size of each one being the number of nodes lying on each $\Gamma_{i}$. Thus, any standard eigensolver for symmetric matrices ( $Q R$, for instance) can be conveniently used for each diagonal block. Since the rank of $\boldsymbol{B}_{11}$ is $2 K$, as a result of the diagonalization we obtain a diagonal matrix of the form

$$
\boldsymbol{D}:=\left(\begin{array}{cc}
\boldsymbol{D}_{11} & \mathbf{0} \\
\mathbf{0} & \mathbf{0}
\end{array}\right) \in \mathbb{R}^{N_{1} \times N_{1}}
$$

where $\boldsymbol{D}_{11}:=\operatorname{diag}\left\{\mu_{1}, \ldots, \mu_{2 K}\right\}$, with $\mu_{j} \neq 0, j=1, \ldots, 2 K$, and an orthogonal matrix $\boldsymbol{Q} \in \mathbb{R}^{N_{1} \times N_{1}}$ such that $\boldsymbol{Q}^{t} \boldsymbol{B}_{11} \boldsymbol{Q}=\boldsymbol{D}$.

Let $\boldsymbol{v}:=\boldsymbol{Q}^{\mathrm{t}} \boldsymbol{u}_{1}$ and $\boldsymbol{S}:=\boldsymbol{Q}^{\mathrm{t}}\left(\boldsymbol{A}_{11}-\boldsymbol{A}_{12} \boldsymbol{A}_{22}^{-1} \boldsymbol{A}_{21}\right) \boldsymbol{Q}$. Then, problem (32) is equivalent to the following one:

$$
\left(\begin{array}{ll}
S_{11} & S_{12} \\
S_{21} & S_{22}
\end{array}\right)\left(\begin{array}{l}
v_{1} \\
v_{2}
\end{array}\right)=\lambda_{h}\left(\begin{array}{cc}
D_{11} & 0 \\
\mathbf{0} & 0
\end{array}\right)\left(\begin{array}{l}
v_{1} \\
v_{2}
\end{array}\right)
$$

Since $S_{22}$ is invertible, the last step consists in eliminating $\boldsymbol{v}_{2}$ from these equations to arrive at

$$
\left(S_{11}-S_{12} S_{22}^{-1} S_{21}\right) v_{1}=\lambda_{h} D_{11} v_{1}
$$

which is a generalized eigenvalue problem of size $2 K$, with both matrices symmetric and $\boldsymbol{D}_{11}$ diagonal and positive definite. Thus, this is a well posed (and small) problem that can be efficiently solved by any standard eigensolver. Finally, the eigenvectors of (31) are easily recovered by successively solving

$$
S_{22} v_{2}=-S_{21} v_{1}, \quad u_{1}=Q v \quad \text { and } \quad A_{22} u_{2}=-A_{21} u_{1} .
$$

\subsection{Adaptive refinement strategy}

For an $h$-finite element adaptive scheme, there are several strategies to determine which elements should be refined. A usual one is the following: all the triangles $T$ with $\eta_{T} \geq \theta \eta_{\mathrm{M}}$ are marked to be refined, where

$$
\eta_{\mathrm{M}}^{2}:=\frac{1}{\# \mathscr{T}_{h}} \sum_{T \in \mathscr{T}_{h}} \eta_{T}^{2}
$$

and $\theta \in(0,1)$ is a parameter which can be arbitrarily chosen.

Our $h p$ adaptive algorithm uses this maximum strategy to mark the triangles to be refined, with the additional consideration that at each step, for each marked triangle, it has to be decided whether to perform a $p$-refinement or an $h$-refinement. In the case of $p$-refinement, the degree $p_{T}$ of the marked element is increased by one and the triangle is kept fixed. On the other hand, in the case of $h$-refinement, the marked element $T$ is subdivided into four triangles, $T=\bigcup_{j=1}^{4} T_{j}^{\prime}$, and the degree is kept fixed in the new elements, i.e., $p_{T_{j}^{\prime}}=p_{T}$. Moreover, the conformity of the mesh is preserved by means of a longest 
edge subdivision strategy on the unrefined neighboring triangles (see [28]). Because of this, it happens that some elements not marked for $h$-refinement, are subdivided anyway into two or three triangles. Thus, in general, we will have that $T=\bigcup_{j=1}^{k} T_{j}^{\prime}$ with $k=2,3$ or 4 .

In order to decide whether to apply a $p$ or an $h$ refinement to a particular triangle, we follow the approach proposed in [16], which is based on the comparison of the current local estimated error with a prediction of this error obtained from the preceding step. If at the preceding step there was an $h$ refinement leading to $T=\bigcup_{j=1}^{k} T_{j}^{\prime}, k=2,3,4$, then the prediction indicator is defined as follows:

$$
\left(\eta_{T_{j}^{\prime}}^{\text {pred }}\right)^{2}:=\gamma_{h}\left(\frac{\left|T_{j}\right|}{|T|}\right)^{p_{T}+1} \eta_{T}^{2},
$$

where $\gamma_{h}$ is a control parameter to be determined. On the other hand, if at the preceding step there was a $p$ refinement on the element $T$, then the prediction indicator is defined by

$$
\left(\eta_{T}^{\text {pred }}\right)^{2}:=\gamma_{p} \eta_{T}^{2}
$$

where $\gamma_{p} \in(0,1)$ is a reduction factor which is chosen arbitrarily. Finally, for elements neither $p$ nor $h$ refined at the preceding step,

$$
\left(\eta_{T}^{\text {pred }}\right)^{2}:=\gamma_{n}\left(\eta_{T}^{\text {pred }}\right)^{2}
$$

where $\gamma_{n}$ is a reduction or amplification factor also arbitrarily chosen. In all cases, we proceed to an $h$ refinement of $T$ when the error indicator $\eta_{T}$ is larger than the prediction indicator $\eta_{T}^{\text {pred }}$ and to a $p$ refinement otherwise.

Altogether, we arrive at the algorithm shown in Table 1.

Table 1: Refinement algorithm

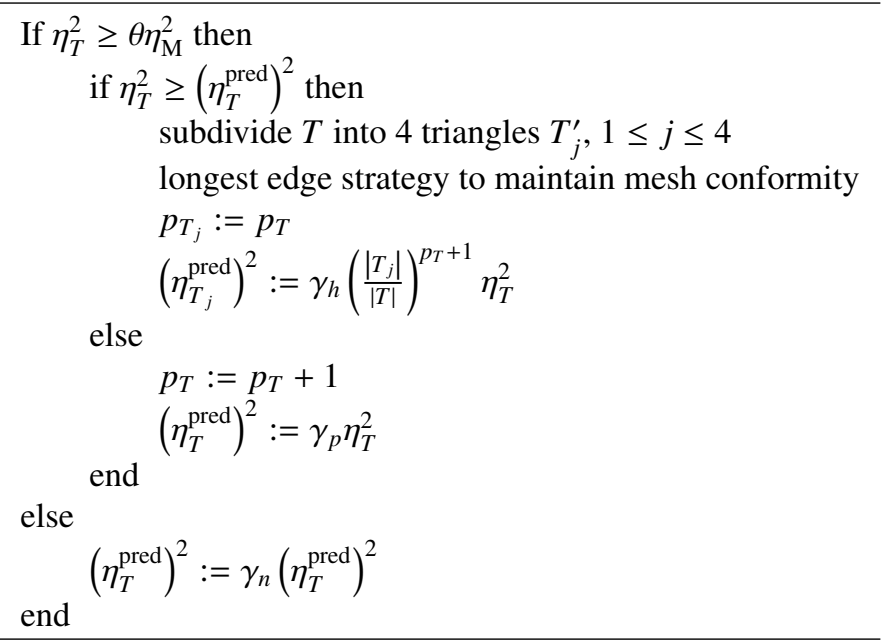

We set $\eta_{T}^{\text {pred }}:=0$ for all elements $T$ on the initial triangulation, so that the first step is a purely $h$-refinement on all elements. Notice that this ensures that no triangle will have vertices lying on two different $\Gamma_{i}$ on subsequent meshes (recall that this is useful for the procedure proposed to solve the generalized eigenvalue problem).

\section{Numerical examples}

We present in this section some numerical results which allow us to assess the performance of the proposed $h p$ adaptive refinement strategy.

In all the numerical examples the control parameters appearing in the algorithm, have been chosen as follows: $\theta=0.75$, $\gamma_{h}=16, \gamma_{p}=0.3$ and $\gamma_{n}=2$.

The color palette, used in the figures, indicates the polynomial degree of each element.

\subsection{Two concentric cylindrical tubes}

In this first test we have taken two concentric cylindrical tubes with inner radio $R_{\mathrm{i}}$ and outer radius $R_{\mathrm{o}}$ (see Figure 2). The analytical solution written in polar coordinates $(r, \phi)$ is as follows:

$$
\begin{gathered}
\lambda_{1}=\lambda_{2}=\frac{1}{\pi} \frac{R_{\mathrm{o}}^{2}-R_{\mathrm{i}}^{2}}{R_{\mathrm{i}}^{2}\left(R_{\mathrm{o}}^{2}+R_{\mathrm{i}}^{2}\right)}, \\
u_{1}=\left(r+\frac{R_{\mathrm{o}}^{2}}{r}\right) \cos \phi, \quad u_{2}=\left(r+\frac{R_{\mathrm{o}}^{2}}{r}\right) \sin \phi .
\end{gathered}
$$

We have taken $R_{\mathrm{i}}=1$ and $R_{\mathrm{o}}=3$, so that the exact eigenvalue is $\lambda \approx 0.2546479$.

The mesh shown in Figure 2 has been used to initiate the adaptive process with quadratic finite elements in all triangles.

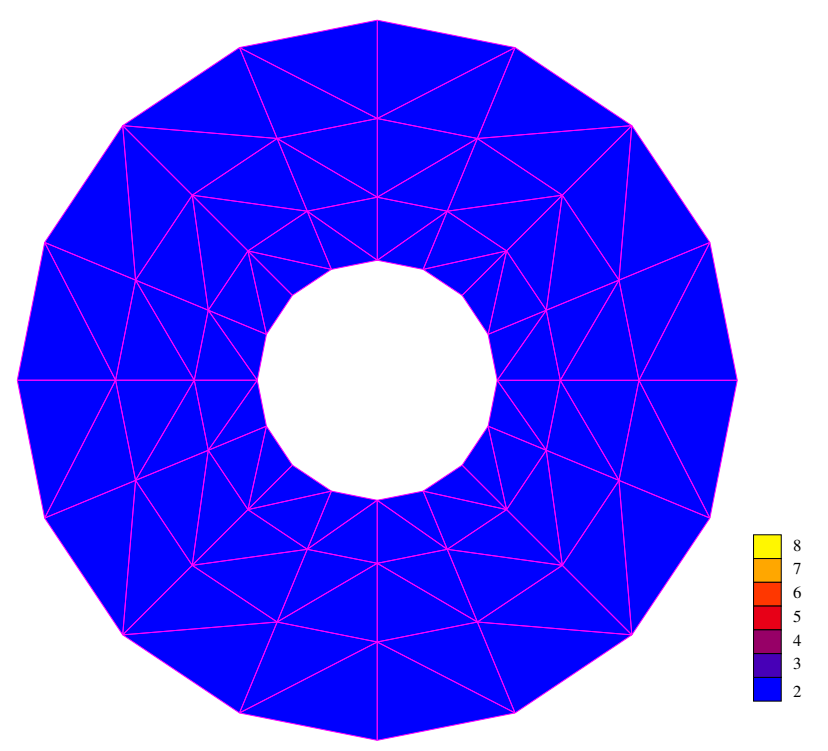

Figure 2: Concentric cylindrical tubes. Domain and initial mesh.

Figure 3 shows the meshes obtained with the adaptive $h p$ algorithm corresponding to steps 7 and 12 of the refinement process. The eigenvalue obtained at this last step with 101,781 degrees of freedom is $\lambda_{h}=0.2546478$.

Since in this test we know the analytical solution, we have used it to compute the so called effectivity indices:

$$
\text { eff }:=\frac{|e|_{H^{1}(\Omega)}}{\eta_{\Omega}} .
$$



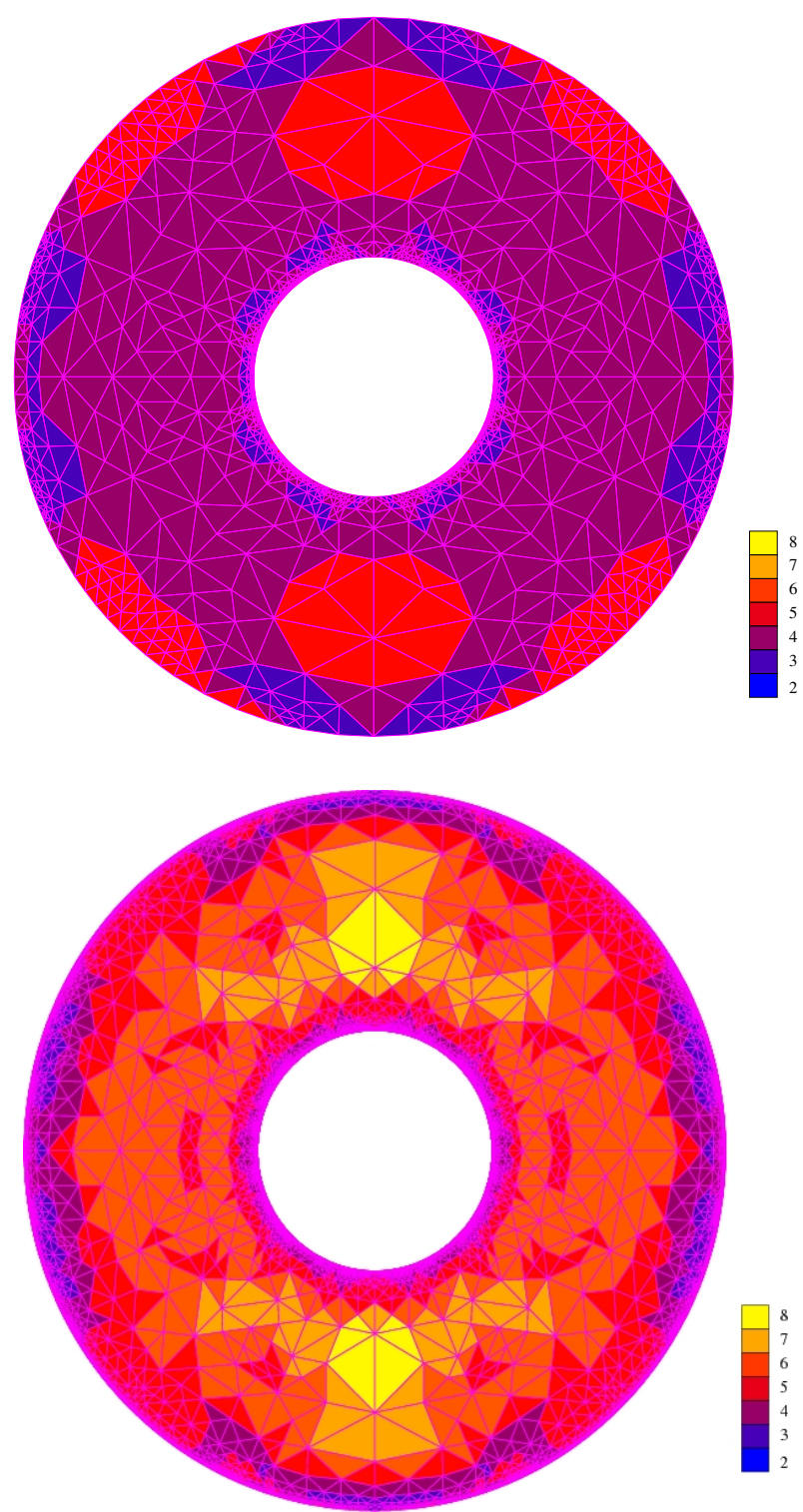

Figure 3: Concentric cylindrical tubes. Refined meshes: steps 7 (top) and 12 (bottom).

We report in Table 2 these indices at all the steps. The table also includes the total number of degrees of freedom $N$ for each step. It can be seen from this table that the effectivity indices remain bounded above and below throughout the refinement process.

\subsection{Rhomboidal tube within a rectangular cavity}

In this test we consider a rhomboidal tube with side $2 \sqrt{2}$ centered in a quadrilateral cavity of side length 8 , as shown in Figure 4.

In this example the fluid domain has reentrant angles at the vertices of the tube. Because of this, the vibration modes involve eigenfunctions which are singular at these four points.

The initial mesh, again with quadratic elements, is shown in Figure 4. Figure 5 shows the mesh at step 22. Figure 6 shows a sequence of zooms of this mesh around one of the reentrant angles.
Table 2: Concentric cylindrical tubes. Effectivity indices

\begin{tabular}{ccc}
\hline Step & $\mathrm{N}$ & eff \\
\hline 0 & 56 & 0.1115 \\
1 & 133 & 0.1350 \\
2 & 247 & 0.1403 \\
3 & 452 & 0.1219 \\
4 & 831 & 0.1419 \\
5 & 1487 & 0.1938 \\
6 & 2945 & 0.2155 \\
7 & 5416 & 0.2621 \\
8 & 10444 & 0.2261 \\
9 & 17815 & 0.2320 \\
10 & 32278 & 0.2259 \\
11 & 56307 & 0.2430 \\
12 & 101781 & 0.2210 \\
\hline
\end{tabular}

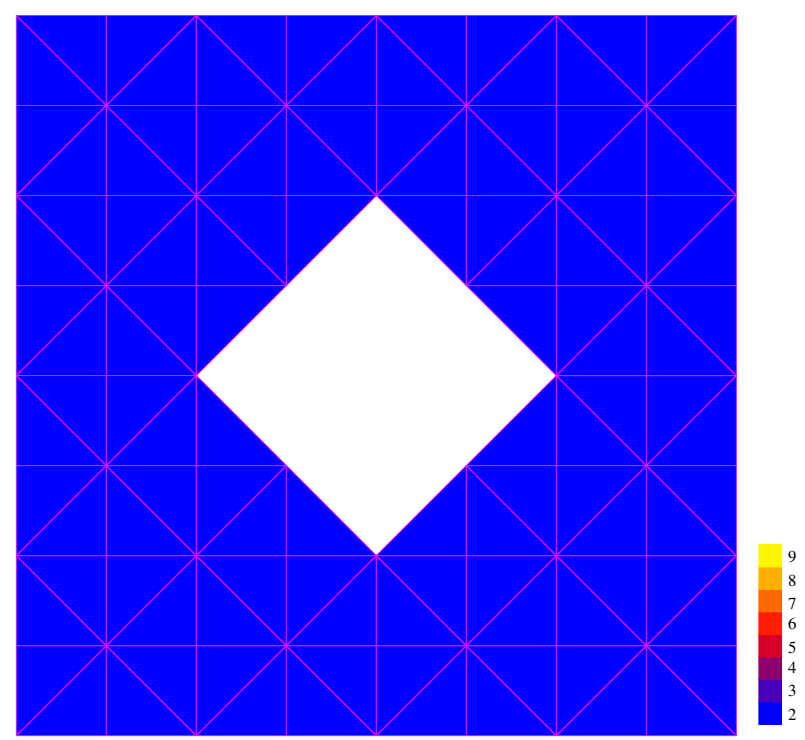

Figure 4: Rhomboidal tube. Domain and initial mesh.

It has been shown in $[29,30]$ that a proper combination of $h$ and $p$ refinement allows to obtaining a rate of convergence

$$
|e|_{H^{1}(\Omega)} \leq C \mathrm{e}^{-\alpha \sqrt{N}},
$$

where $N$ is the number of degrees of freedom in the finite element approximation. Figure 7 shows a plot of $\log \eta_{\Omega}$ versus $\sqrt{N}$, which shows that the estimated error $\eta_{\Omega}$ attains such an exponential rate in this problem.

No analytical solution is available in this case to verify if the actual error also attains an exponential rate of convergence. To provide some numerical evidence of such a behavior, we have estimated the error of the computed eigenvalues by using as 'exact' a more accurate approximation obtained by an extrapolation procedure. To do this, we have used the fact that the computed eigenvalues are expected to converge with a double order and we have determined the parameters $\lambda, \kappa$ and $\alpha$ in the 


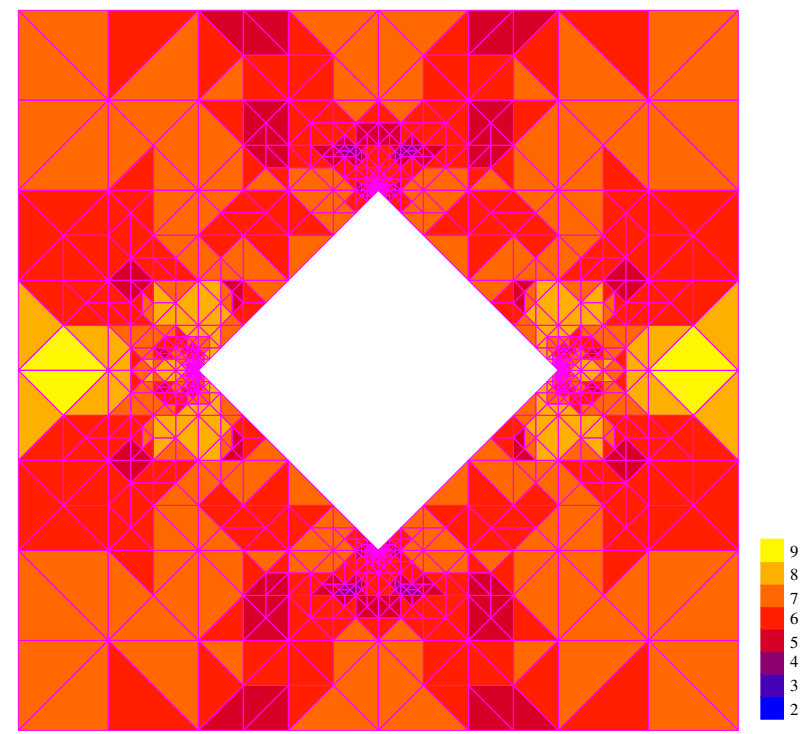

Figure 5: Rhomboidal tube. Refined mesh: step 22.

model

$$
\lambda_{h}=\lambda+\kappa \mathrm{e}^{-2 \alpha \sqrt{N}},
$$

by means of a weighted least-squares fitting. The weights have been chosen so that the most precise computed values $\lambda_{h}$ play the more significant role in the fitting. Thus, we have obtained a value $\lambda=0.07896$, which we have used to plot $\log \left|\lambda_{h}-\lambda\right|$ versus $N^{1 / 2}$. This plot is shown in Figure 8, where a linear dependence can be clearly seen for sufficiently large values of $N$. This is coherent with the expected exponential decay of the error with respect to the number of degrees of freedom.

\subsection{A bundle of quadrilateral tubes}

In this last example we have computed the main vibration mode (i.e., the mode with smallest eigenvalue) of a system closer to the actual applications: five square tubes immersed in a fluid occupying a rectangular cavity as shown in Figure 9.

Figure 10 shows the mesh obtained after 8 steps of the $h p$ adaptive scheme. Figure 11 shows the fluid velocity field computed from the pressure obtained at the last step. The arrows at the center of each tube show the directions of the tubes motion.

\section{Conclusions}

An $h p$ finite element method has been proposed to compute the free vibrations of a bundle of tubes immersed in an incompressible fluid contained in a rigid cavity. Convergence and a-priori error estimates have been obtained for the $h p$-finite element approximation of this spectral problem.

An a-posteriori error indicator has been proposed and its reliability and efficiency have been rigorously proved. We have introduced an adaptive algorithm based on this indicator, which allows refining some of the elements and increasing the polynomial degree in others at each step.
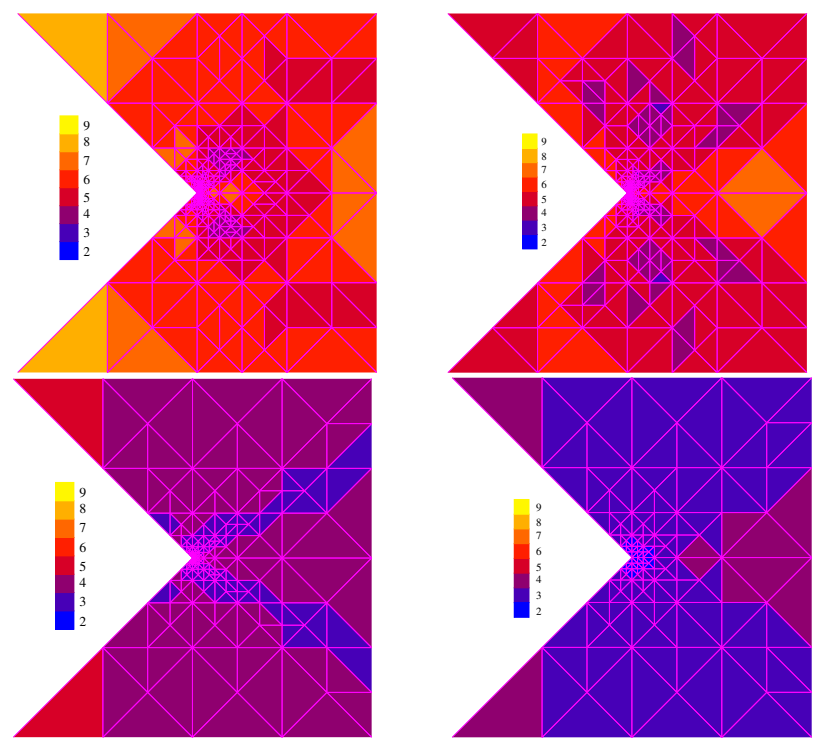

Figure 6: Rhomboidal tube. Refined mesh: step 22. Successive zooms

The reported numerical experiments for different cavities and different shapes of tubes show the good performance of the error indicator and the adaptive scheme. Numerical evidence of the theoretically expected exponential convergence is also reported.

\section{Acknowledgement}

This work was partially supported by ANPCyT under grant PICT 2006-01307. The first author was partially supported by ANPCyT under grant PICT-2007-00910 and by Universidad de Buenos Aires under grant X007. The first and second authors are members of CONICET, Argentina. The third author was partially supported by FONDAP and BASAL projects, CMM, Universidad de Chile.

\section{References}

[1] M. G. Armentano, The effect of reduced integration in the Steklov eigenvalue problem, M2AN Math. Model. Numer. Anal. 38 (2004) 27-36.

[2] A. Bermúdez, R. Durán, R. Rodríguez, Finite element solution of incompressible fluid-structure vibration problems, Internat. J. Numer. Methods Engrg. 40 (1997) 1435-1448.

[3] A. Bermúdez, R. Rodríguez, D. Santamarina, A finite element solution of an added mass formulation for coupled fluid-solid vibrations, Numer. Math. 87 (2000) 201-227.

[4] C. Conca, A. Osses, J. Planchard, Asymptotic analysis relating spectral models in fluid-solid vibrations, SIAM J. Numer. Anal. 35 (1998) 10201048.

[5] C. Conca, J. Planchard, M. Vanninathan, Fluid and Periodic Structures, Masson, Paris, 1995.

[6] H. J. P. Morand, R. Ohayon, Fluid-Structure Interaction, John Wiley \& Sons, Chichester, 1995.

[7] J. Planchard, Eigenfrequencies of a tube bundle placed in a confined fluid, Comput. Methods Appl. Mech. Engrg. 30 (1983) 75-93.

[8] J. Planchard, M. Ibnou-Zahir, Natural frequencies of tube bundle in an uncompressible fluid, Comput. Methods Appl. Mech. Engrg. 41 (1983) 47-68. 


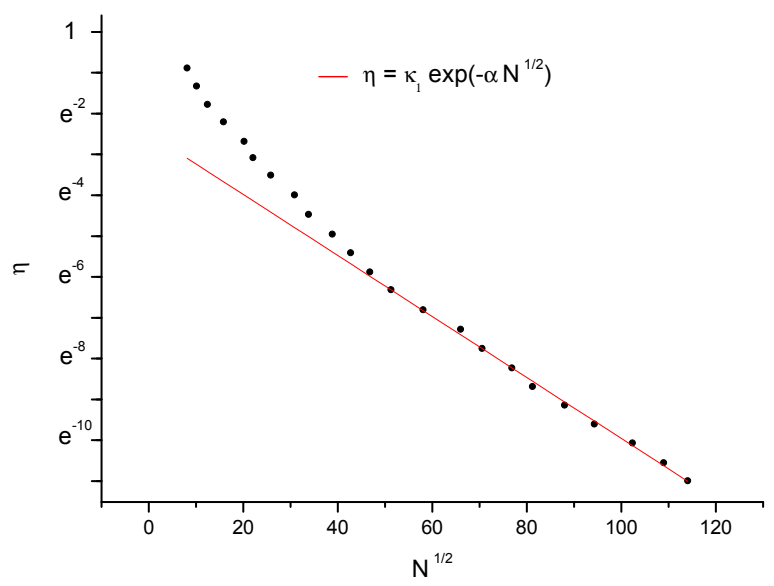

Figure 7: Rhomboidal tube. Estimated error curve.

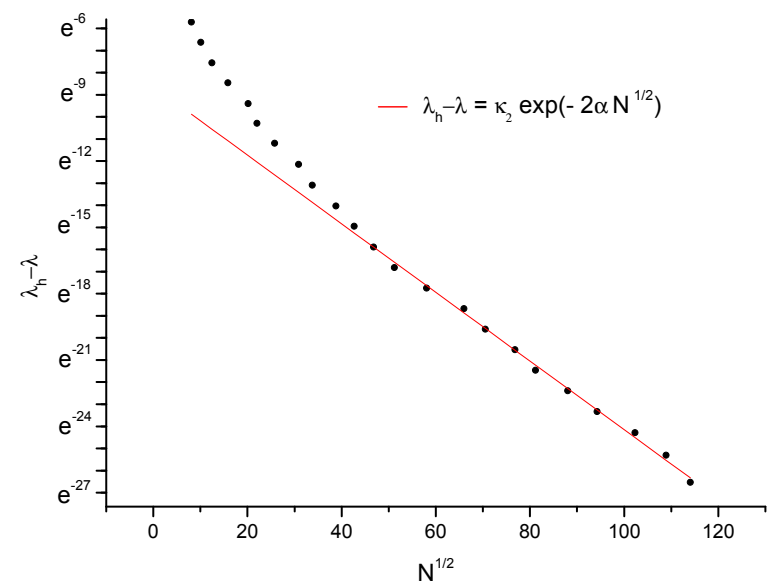

Figure 8: Rhomboidal tube. Error curve for the eigenvalue.

[9] M. G. Armentano, C. Padra, A posteriori error estimates for the Steklov eigenvalue problem, Appl. Numer. Math. 58 (2008) 593-601.

[10] R. G. Durán, C. Padra, R. Rodríguez, A posteriori error estimates for the finite element approximation of eigenvalue problems, Math. Models Methods Appl. Sci. 13 (2003) 1219-1229.

[11] R. G. Durán, L. Gastaldi, C. Padra, A posteriori error estimators for mixed approximations of eigenvalue problems, Math. Models Methods Appl. Sci. 9 (1999) 1165-1178.

[12] M. G. Larson, A posteriori and a priori error analysis for finite element approximations of self-adjoint elliptic eigenvalue problems, SIAM J. Numer. Anal. 38 (2000) 608-625.

[13] C. Lovadina, M. Lyly, R. Stenberg, A posteriori estimates for the Stokes eigenvalue problem, Numer. Methods PDEs 25 (2009) 244-257.

[14] M. Ainsworth, B. Senior, Aspects of an adaptive $h p$ finite element method: Adaptive strategy, conforming approximation and efficient solvers, Comput. Methods Appl. Mech. Engrg. 150 (1997) 65-87.

[15] M. Ainsworth, B. Senior, An adaptive refinement strategy for $h p$-finite element computations, Appl. Numer. Math. 26 (1998) 165-178.

[16] J. M. Melenk, B. I. Wohlmuth, On residual-based a posteriori error estimation in $h p$-FEM, Adv. Comput. Math. 15 (2001) 311-331.

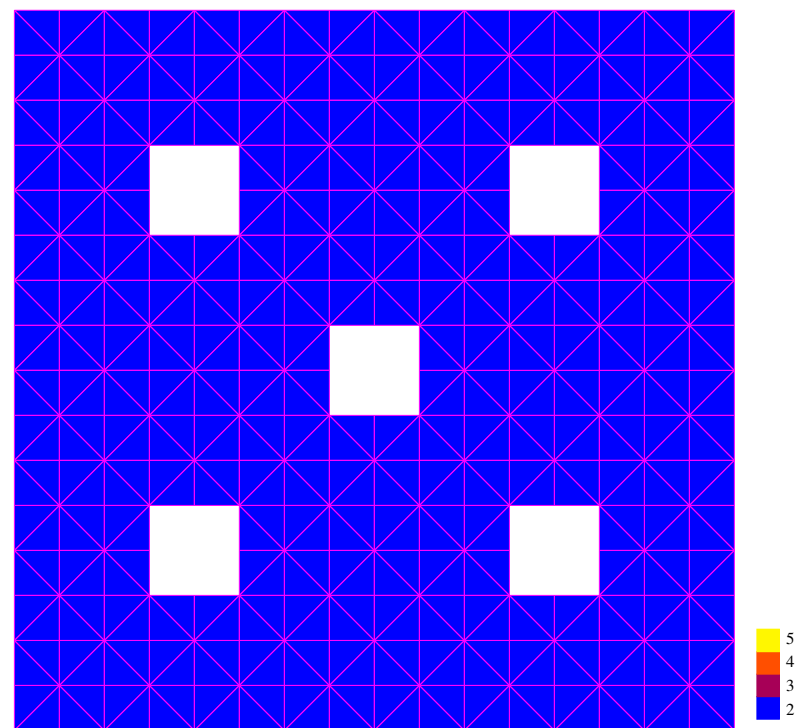

Figure 9: Bundle of quadrilateral tubes. Domain and initial mesh.

[17] J. E. Tarancon, F. J. Fuenmayor, L. Baeza, An a posteriori error estimator for the $p$ - and $h p$-versions of the finite element method, Internat. $\mathrm{J}$. Numer. Methods Engrg. 62 (2005) 1-18.

[18] D. Boffi, M. Costabel, M. Dauge, L. Demkowicz, Discrete compactness for the $h p$ version of rectangular edge finite elements, SIAM J. Numer. Anal. 44 (2006) 979-1004.

[19] D. Boffi, Approximation of eigenvalues in mixed form, discrete compactness property, and application to $h p$ mixed finite elements, Comput. Methods Appl. Mech. Engrg. 196 (2007) 3672-368.

[20] M. Azaiez, M. O. Deville, R. Gruber, E. H. Mund, A new $h p$ method for the -grad(div) operator in non-Cartesian geometries, Appl. Numer. Math. 58 (2008) 985-998.

[21] R. Hiptmair, P. D. Ledger, Computation of resonant modes for axisymmetric maxwell cavities using $h p$-version edge finite elements, Internat. J. Numer. Methods Engrg. 62 (2005) 1652-1676.

[22] J. Coyle, P. D. Ledger, Evidence of exponential convergence in the computation of maxwell eigenvalues, Comput. Methods Appl. Mech. Engrg. 194 (2005) 587-604.

[23] P. D. Ledger, K. Morgan, The application of the $h p$-finite element method to electromagnetic problems, Arch. Comput. Methods Engrg. 12 (2005) 235-302.

[24] I. Babuška, J. Osborn, Eigenvalue Problems, in: P. Ciarlet, J. Lions (Eds.), Handbook of Numerical Analysis, vol. II, North-Holland, Amsterdam, 1991, pp. 641-787.

[25] P. Grisvard, Elliptic Problems in Nonsmooth Domain, Pitman, Boston, 1985.

[26] I. Babuška, M. Suri, The $p$ and $h-p$ versions of the finite element method, basic principles and properties, SIAM Review 36 (1994) 578-632.

[27] P. A. Raviart, J. M. Thomas, Introduction à l'Analyse Numérique des Equations aux Dérivées Partielles, Masson, Paris, 1983.

[28] R. Verfürth, A Review of A Posteriori Error Estimation and Adaptive Mesh-refinement Techniques, Wiley \& Teubner, 1996.

[29] B. Q. Guo, I. Babuška, The $h$ - $p$ version of the finite element method. Part 1: The basic approximation results, Comput. Mech. 1 (1986) 21-41.

[30] B. Q. Guo, I. Babuška, The $h-p$ version of the finite element method. Part 2: General results and applications, Comput. Mech. 1 (1986) 203-220. 


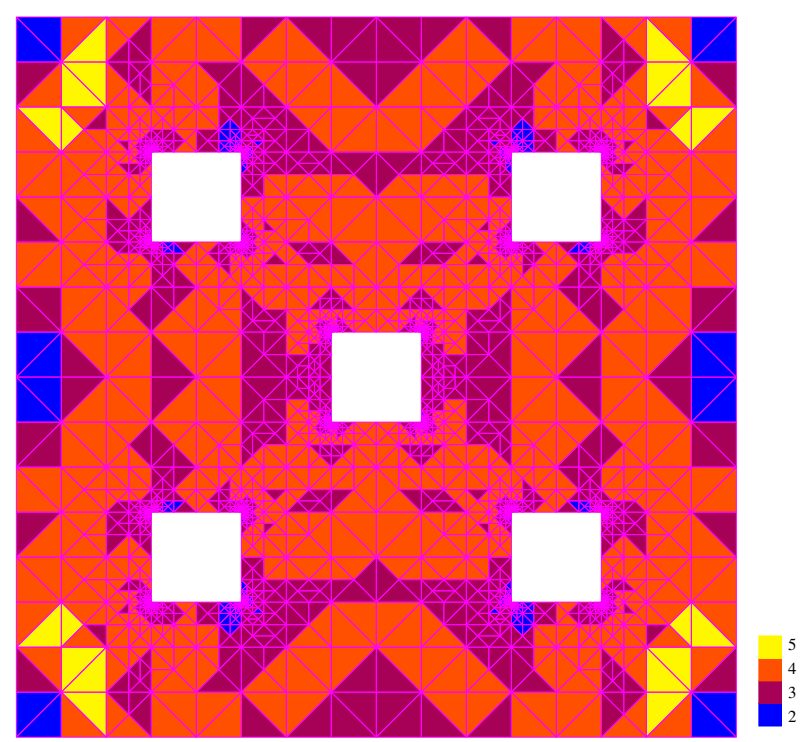

Figure 10: Bundle of quadrilateral tubes. Mesh at step 8

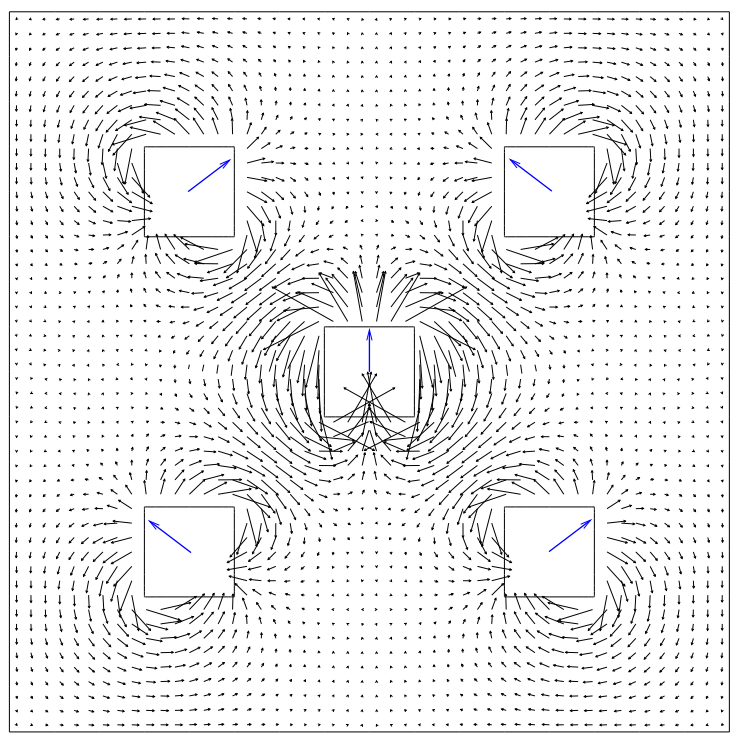

Figure 11: Bundle of quadrilateral tubes. Computed fluid and tubes velocities. 